\title{
ETIOLOGICAL FACTORS IN THE PRODUCTION OF COMPLETE RECTAL PROLAPSE
}

\author{
By I. P. Todn, M.S., M.D., F.R.C.S., D.C.H. \\ Surgeon, The Royal Hospital of St. Bartholomerc, London, and St. Mark's Hospital, London
}

Complete rectal prolapse is not a rare condition but it is one which may often defy attempts at surgical cure. One has only to find that more than 60 operations have been devised in an equal number of years, to realize that this is true. The etiology of the condition is not really understood and it is probable that the prolapse is merely part of a symptom complex.

The only lesion known to cause complete rectal prolapse is complete section of the cauda equina. When it occurs in this condition, it will be found that the care of bowel function has been neglected for some time. Prolapse can be reproduced in the dog by cauda equina section.

In order, therefore, to understand the factors of etiological significance present in a case of idiopathic complete rectal prolapse, it would seem appropriate to describe initially the changes which occur in:-

(I) Cauda equina section where prolapse may occur.

(2) Low spinal cord section where prolapse does NOT occur.

It is necessary first, however, to refresh our memory of the normal physiology of defaecation, and then to consider those factors which appear to have a bearing on the production of prolapse, keeping in mind the neurological sequela.

When the rectum is distended to a certain size, it contracts, raising the pressure upon the rectal wall and faecal mass to provoke a conscious (or possibly subconscious) sensory response-the call to stool. This may be voluntarily refused or answered, but whichever it is, as the rectal distension-contraction increases, the internal sphincter tone is lessened. If the call is answered a coordinated voluntary muscular response, the strain to stool, occurs. The breath is held, the diaphragm descends, the abdominal wall is pulled in and the levator diaphragm contracts to become more horizontal and resists a downward thrust. Pro- vided there is no local obstruction, the stool is passed.

Voluntary external sphincter contraction only occurs if the call to stool is refused. The contraction is, and can be, maintained for a short period only, during which plastic adaptation, a fall in rectal tone and regaining of internal sphincter tone, occurs. Thus involuntary control is regained.

In transection of the cauda equina, this normal train of events does not occur. The effect of the lesion upon the lower limbs need not concern us here. However, the abdominal wall musculature remains normal, though the levator ani diaphragm and external sphincter muscle are paralysed and atonic. The call to stool (rectal sensation) is lost. As a result of these changes, the anus becomes relatively patulous with no anal reflex as the reflex arc is interrupted; the rectum itself is inert and a very large quantity of faeces is required to produce even slight rectal contraction, sluggish internal sphincter relaxation but no stimulation of consciousness. Automatic defaecation, for such is the term given (Denny-Brown and Robertson, 1935), could theoretically occur as the result of the rectal contraction, but it is almost completely ineffectual. The normal straining mechanism is available but co-ordination of effort is difficult as sensation has been lost. Furthermore, straining may be misdirected because pelvic floor resistance is gone.

Rectal prolapse is very rare following any other cord transection and does not occur when the abdominal wall is paralysed. In these lesions the anal reflex arc is intact, the anus is less patulous as some tone is present in the external sphincter, and the anal reflex itself is present. In these cases reflex defaecation may occur and though this is a poor substitute for the normal, rectal contraction is induced by a smaller faecal volume than in the cauda equina lesion and internal sphincter relaxation is more complete and less sluggish. Reflex 


\section{NORMAL DEFAECATION}

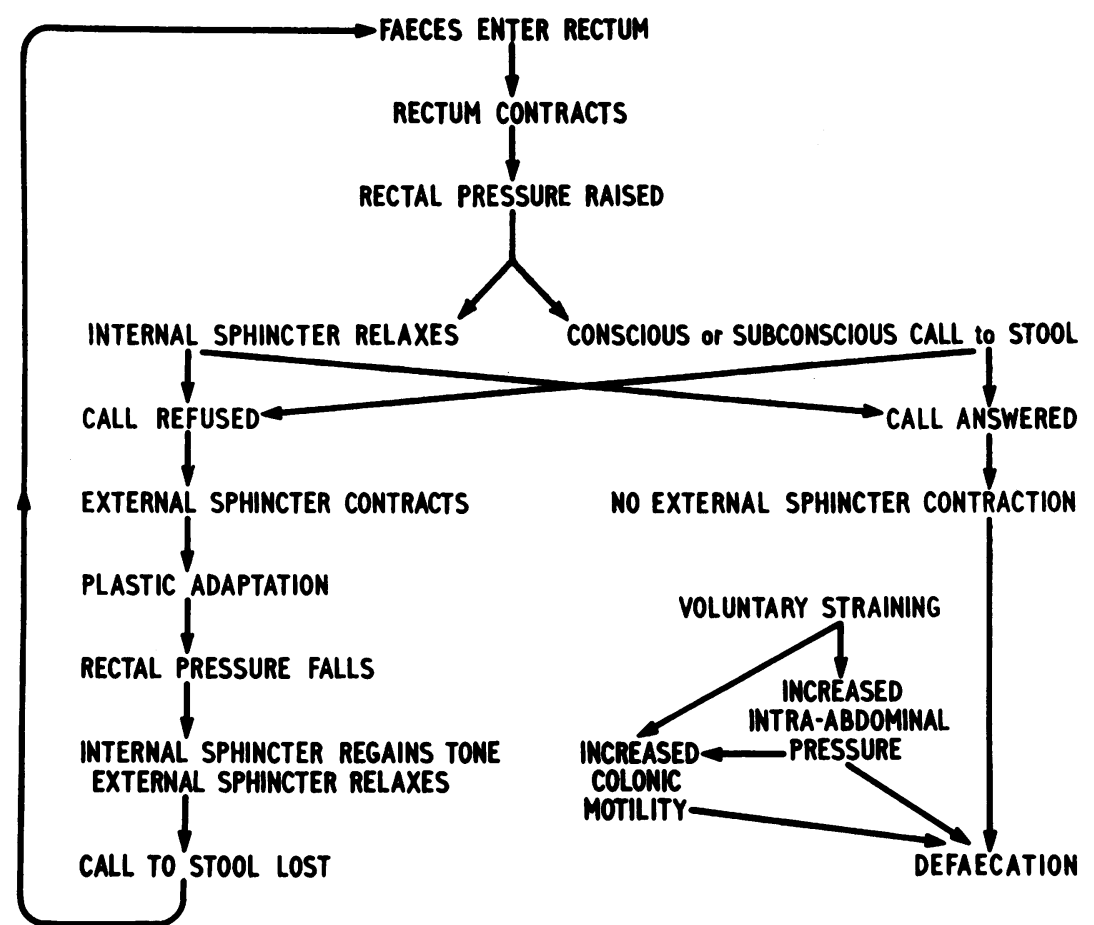

defaecation can be augmented by perineal irritation (e.g., scratching). Even though rectal sensation is lost, voluntary straining is not possible, for the abdominal muscles are paralysed and thus the effort cannot be misdirected.

In idiopathic complete rectal prolapse, rectal sensation is usually reduced and inadequate. The loss would appear to be in the rectal wall itself, for these cases seldom show evidence of any neurological abnormality. Thus a larger volume distending the rectum is needed to produce any rectal contraction; consciousness is only poorly stimulated but the internal sphincter is caused to relax. These facts are easily demonstrated by inserting a balloon, attached to a sphygmomanometer, into the lower $5-7 \mathrm{~cm}$. of the rectum, Goligher's sensitive area (Goligher, 195I), and inflating it slowly with known volumes. In the normal subject a moderate volume (200-250 c.c.) exerts a pressure of $20-40 \mathrm{~mm}$. of mercury upon the rectal wall, causing an increase in rectal tone and, thus, sensation. Normally, co-ordinating with the sensation, voluntary straining, correctly resisted by the pelvic floor, directs the faeces out of the anus. In rectal prolapse the volume is higher and the pressure to provoke contraction

needs to be increased $(60-80 \mathrm{~mm}$.). As the call to stool is less definite, straining may not always be $\stackrel{\square}{\unrhd}$ carried out at the most appropriate time. The $\overrightarrow{\vec{A}}$ prolonged neglect of answering the call to stool $\frac{3}{3}$ sets up a vicious circle, larger and larger volumes being required to provoke a sensory response. This, too, is a factor in the cauda equina case. Plastic adaptation is complete but internal sphinc- 3 . ter relaxation is extreme. Thus, we can see that overdistension causes a less satisfactory rectal. response and, if the call is frequently ignored, $\stackrel{\circ}{\circ}$ dyschezic constipation results. Re-education of this type of bowel is difficult. Whether this is the mechanism by which sensation is diminished in $D$ complete rectal prolapse one cannot be sure, but there is frequently a history of prolonged constipa- $N$ tion or inadequate frequent bowel-actions which the patient may call diarrhoea. The fact, however, $N$ remains, sensation is diminished in the rectal $\mathbb{N}_{\mathrm{N}}$ prolapse.

The anus in complete rectal prolapse is patu-co lous, though an anal reflex is present. Rectal dis- $\bar{\varnothing}$ tension causes internal sphincter relaxation. If $\stackrel{\mathcal{P}}{+}$ the distension is prolonged, internal sphincter ${ }^{\circ}$ relaxation is also prolonged. External sphincter $\frac{0}{\vec{D}}$

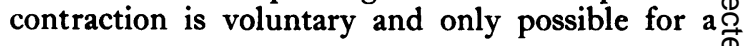


short duration. According to Gaston (Gaston, I948), its fatigue time is about 52 sec. Once prolapse has occurred through the relaxed anus, a stretched patulous anus is irrevocable.

The presence of a poor levator diaphragm in perineal and rectal prolapse has long been known. Muir (1955) drew attention to this, and his work and that of Berglas and Rubin (1953) showed the downward angulation and sagging of the normally more or less horizontal diaphragm, the levator ani muscles. A clinical feature of rectal prolapse which has not been stressed, however, is the divarication of the pubococcygeal portions of the levator ani and the relative absence of the puborectalis muscles. This factor causes the normal anorectal ring to be inconspicuous and only palpable when the patient contracts the levator voluntarily. In the perineal phase of an abdominoperineal excision of the rectum, it is easy to see that the puborectalis fuses with the longitudinal muscle of the bowel whilst the pubococcygei encircle it posterolaterally. If, however, the levator floor is approached from the perineum in an attempt to reinforce the muscular diaphragm in a case of rectal prolapse, no puborectalis attachment is found to the rectum and the pubococcygei are widely separated bands. This divarication of muscles allows the rectum to move backwards to leave a potential gap between rectum and vagina. It is into this space that herniation of gut contents takes place. The perineal hernia with its sac of peritoneum, the deepened pouch of Douglas, descends, is partly obstructed initially by the perineal body, and pushes itself into the anterior rectal wall, the first portion of the prolapse to be seen. If this anterior gap is blocked with a finger in the rectum, no prolapse takes place. It must be remembered when devising a suitable operation, that this gap should be filled by the rectum, and the levator muscles approximated, and attached, behind it, or the posterior hiatus filled in some other way.

It is not known why these changes occur in the pelvic floor, in complete rectal prolapse, nor why there is often a lack of supporting fat. They are, however, almost identical with those changes seen when the pelvic floor is paralysed.

It is possible that there are congenital abnormalities of rectal attachment and of the pelvic diaphragm in cases of rectal prolapse. With the abdominal approach one often finds a deep pouch of Douglas, possibly due to failure of peritoneal fusion to form the fascia of Denonvilliers, together with a mesorectum. It is possible that the puborectalis and pubococcygei are abnormal, too, though this factor probably is partially acquired. Misdirected straining would exaggerate any abnormality of the pelvic diaphragm. A lack of co- ordination between the effort of straining and rectal distension, sensation being impaired, will cause an increase in abdominal pressure which will be most apparent at the weakest spot, the bottom of the rectovaginal pouch.

There is no abnormality of the bony pelvis or lumbar curve in these cases which could potentially throw more strain on the pelvic diaphragm.

There are several additional factors which throw light on the part played by lack of sensation, the effect of straining and so on in the production of rectal prolapse.

Simple rectal prolapse occurs frequently in children. They are often made to sit on a pot by over-zealous parents for long periods. The parents do not know whether the rectum is full and the child may not yet appreciate the fact either; but it is encouraged to strain. It may strain with the bowel empty, and thus inco-ordinate the effort. This is not the only instance when straining with an empty bowel produces prolapse. Wright (1949) has told of a peculiar sect of people from Eastern Europe who develop rectal prolapse as a result of straining with the rectum empty, practised as a religious fetish.

There is a very high incidence of complete prolapse amongst mental defectives. This is probably associated with bowel neglect either as the result of sensory misinterpretation, failure to heed the normal call to stool or the result of inadequate bowel care and unsuitable purgation by attendants. There is thus, either a failure to coordinate straining with the sensory stimulus, or a failure of registration and interpretation of the stimulus in these cases. Leucotomized persons also may produce rectal prolapse, probably again a failure to interpret and answer the call to stool. Rarely the condition has been seen associated with disseminated sclerosis and tabes dorsalis and, knowing the diffuse nature of these diseases, it seems possible that the neurological lesion may be causative in these cases.

Complete division of either the internal or external sphincter muscle alone does not cause rectal prolapse. Division of both may do so. This implies that normal sphincteric tonus is adequate to prevent prolapse provided that other factors are not present.

There is one other facet of the disease which is difficult to assess. Though rectal prolapse occurs equally in both sexes, in the $25-35$ age-group, it is far more frequent in the older female. In this group spinsters proportionately far outnumber married women. Childbirth hastens the onset of symptoms, but does not appear to cause the condition. Perhaps the congenital factor mentioned may predispose in these people. Many, however, have commented on the odd mental 
make-up of these cases and Thompson (1958) has suggested that rectal straining and production of prolapse may be a form of masturbation.

\section{Summary and Conclusions}

The etiology of complete rectal prolapse is unknown. Certain neurological factors give pointers as to its mode of production, as the disease is seen in patients with a cauda equina lesion where bowel care is neglected, and more rarely in cases of tabes dorsalis and disseminated sclerosis. Sensory appreciation is faulty, either as a result of poor interpretation of the normal stimulus, as demonstrated by the increased incidence of the disease in the mentally deficient or leucotomized persons, or as a result of a rectal wall abnormality with an associated hypoexcitability. The patulous anus is the result of prolonged stretching and excessive rectal distension which lead to an atonic external and internal sphincter. A straining mechanism, usually the abdominal wall musculature, must be intact, but a lack of co-ordination exists between the appreciation of the full-rectum stimulus and straining.

In addition, a congenital factor, the presence of a mesorectum and a deep rectovaginal (recto- vesical) pouch is apparent, and this is partly suggested by the preponderance in spinsters. The reason for failure of the levator diaphragm is not known though a congenital effect may be present here also.

A logical surgical approach would seem to be to deal with those factors which are understood. The bowel may be shortened and made to adhere to the sacral curve, the bottom of the pouch of Douglas may be elevated, but, above all, the rectum should be pushed forward and a new anorectal ring formed posterior to it. The anal canal may then angulate backwards in its normal manner.

Plication operations to close the anus are illogical. The anus must be held as near shut as possible so that sphincteric tone may return. A Thiersch operation would seem the most logical 'sphincteroplasty'.

\section{BIBLIOGRAPHY}

BERGLAS, B., and RUBIN, I. C. (1953), Surg. Gynec. Obstet. $97,277,677$.

DENNY-BROWN, D., and ROBERTSON, E. G. (1935), Brain, 58,256 .

GASTON, E. A. (1948), Surg. Gynec. Obstet., 87, 280, 669. GOLIGHER, J. C. (1951), Ann. roy. Coll. Surg. Engl., 8, 421. MUIR, E. G. (1955), Proc. roy. Soc. Med., 48, r, 33. THOMPSON, H. R. (1958), personal communication. WRIGHT, A. D. (1949), Proc. roy. Soc. Med., 42, 1005.

\section{E. \& S. LIVINGSTONE, LTD}

SURGERY OF THE ANUS, ANAL CANAL AND RECTUM

By E. S. R. HUGHES, M.D.(Melb.), M.S.(Melb.), F.R.C.S.(Eng.), F.R.A.C.S.

316 pages. $\quad 398$ illustrations.

50 s.

"Well written, replete with illustrations clear and valuable, and full of practical instruction for those who are engaged in this speciality." Proceedings of the Royal Society of Medicine.

\section{SURGERY OF THE CAECUM AND COLON} BY STANLEY AYLETT, M.B.E., F.R.C.S.

303 pages. $\quad 170$ illustrations.

"Mr. Aylett will earn the gratitude of a succession of surgeons for the exposition of the principles and details of treatment made so clearly and logically. Full of interest and help to the surgeon of experience, this monograph will be a continuing boon to the young surgeon."-Australian and New Zealand Journal of Surgery.

\section{CANCER OF THE RECTUM}

Edited by CUTHBERT DUKES, O.B.E., M.D., M.Sc., F.R.C.S. Fully illustrated. In active preparation. The third volume in the new series of monographs entitled "Neoplastic Disease at Various Sites" under the general editorship of Professor D. W. Smithers. The reputation of the editor is unparalleled and the work will become the most up-to-date and authoritative source of knowledge in this field.
SURGERY OF THE COLON

By E. S. R. HUGHES.

420 pages. 234 illustrations. About 60 s.

This companion volume is a practical book. It will enhance the author's reputation for lucid, succinct writing allied with clear and carefully chosen illustrations.

\section{ULCERATIVE COLITIS AND ITS SURGICAL TREATMENT}

By BRYAN N. BROOKE, M.D., M.Chir., F.R.C.S. I58 pages. III illustrations. 37s. 6d. " Mr. Brooke states his case clearly and fairly. The beautiful illustrations, many of which are coloured, are excellently produced. Every surgeon should certainly read this book, and the wise surgeon will buy a copy."-Lancet.

\section{HISTOLOGICAL APPEARANCES OF}

\section{TUMOURS}

By R. WINSTON EVANS, T.D., B.Sc., M.R.C.S., L.R.C.P.

789 pages.

989 illustrations.

90 s.

" This remarkable book is a vivid, accurate and most informative account of the structure of tumours, and to the very adequate description is added a vast amount of interesting and relevant material which completes the story. A first-class achievement."-Journal of the Royal College of Surgeons of Edinburgh. 